\title{
Spatiotemporal Parameters of Gait in Filipino Adults Using the 3-D Motion Capture System
}

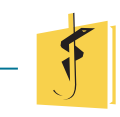

\author{
Maria Belinda Cristina C. Fidela, MD, Consuelo B. Gonzalez- \\ Suarez ${ }^{b}, M D, P h D$, Angelo R. dela Cruz , PhD, Edison A. Roxas ${ }^{c}$, \\ Ma. Roxanne Fernandez ${ }^{a}$, MSPT, Christopher Gabon Cruz ${ }^{a}$
}

\section{ABSTRACT}

Background and Purpose: Gait is one of the outcome measures used in evaluation in the field of rehabilitation and there is a need for reference data of gait parameters primarily to understand the physiological significance of these parameters, describe their changes in pathologic gait for better understanding of pathophysiology and be able to provide the appropriate therapeutic approach. The purpose of this study was: 1) To derive a reference data of spatiotemporal parameters of gait among Filipinos, which include step time, stride time, stance time, swing time, single limb support time, double limb support time, cadence, speed, step length, stride length, and step width; 2) To determine correlation of age, gender, and anthropometric measures with spatiotemporal parameters, and 3) To determine gait symmetry.

Materials and Methods: In this cross-sectional study, 374 participants aged 20-69 years (males = 181 , females $=193$ ) were included. They were asked to walk in their comfortable speed. Spatiotemporal parameters were obtained using the Vicon motion capture system.

Maria Belinda Cristina C. Fidel

begsmd@yahoo.com.ph

a. College of Rehabilitation Sciences, University of Santo Tomas

b. Faculty of Medicine and Surgery, University of Santo Tomas

c. Electronics Engineering Department, Faculty of Engineering, University of Santo Tomas
Results: The walking speed, step length, stride length, and step width declined with age. Filipino men demonstrated higher values in all parameters except cadence which is higher in Filipino women. Height and leg length both have positive correlation with all parameters except for cadence. Weight has a positive correlation with all spatiotemporal parameters except for cadence, swing time, single limb support time, and speed. Cadence and stride length were symmetrical between right and left lower extremities.

Conclusion: Spatiotemporal parameters among healthy Filipinos 20-69 years old were presented including their correlations with age, gender, and anthropometric measures. This can serve as a reference for future studies in gait where Filipinos are the participants.

Key words: anthropometric, gait analysis, reference values, spatiotemporal parameters

\section{INTRODUCTION}

An essential requirement for a satisfactory quality of life is ambulation.[1] The most commonly reported measurements of gait are the spatiotemporal parameters which include walking speed, stride time and length, step time and length, as well as the durations of stance phase and swing phase.[2] Aside from spatiotemporal parameters, gait symmetry is another measure gaining acceptance and used as an indicator 
of fall risk, fear of falling, and the capacity for community ambulation. [3]

Gait analysis provides important information on the functional capacity of patients when making disability assessments and treatment planning.[4] One of its main tasks is to determine whether a motion is physiological or if it differs, how much it differs from normal values.[5] The aim of rehabilitation is to improve function, therefore, a reference data of gait parameters is warranted.[2]

Studies on gait have been primarily undertaken in Western countries, ie, United States, France, and Italy with a few studies performed on Korean nationalities investigating normal gait patterns. These studies have shown that spatiotemporal gait parameters are affected by culture as well.[6]

To date there is no reference data on gait parameters among Filipinos. This paper reports on the reference data of normal Filipino gait pattern using the 3D motion capture system. It also determined the correlation of age, gender, and anthropometric measures (height, weight, body mass index, true leg length) with the different spatiotemporal parameters of gait (step time, stride time, stance time, swing time, single limb support time, double limb support time, cadence, speed, step length, stride length, and step width). Lastly, it determined the symmetry pattern of spatiotemporal parameters between the right and left lower extremity during gait.

\section{METHODOLOGY}

\section{Study Design and Subjects}

A cross sectional study was performed. All the subjects understood the purpose of this study and gave their written and informed consent prior to their participation. The study protocol was approved by the Ethical Review Committee and complied with the ethical principles of the Declaration of Helsinki.

Participants were recruited from the barangays of the city of Manila. With a total population of our city of $1,642,743$, sample size of at least 372 will be needed to obtain a confidence level of $95 \%$ and an alpha level of 0.05 . The sample was divided into males and females with three age categories per gender 20-39 years old as young adults (YA), 40-59 years old as middle adults (MA), and 60-69 years old as young old ( $\mathrm{YO}$ ), based on the physical development stage in adulthood where young adults (20-39 years old) would have peak of physical abilities, middle adults (40-59 years old) would have gradual physical decline and young old $1>60$ years old) would have further decrease in reaction time as well as diminished muscle strength.

They filled out the data sheet for their demographic data and were examined by the physician of the research team to determine their eligibility for the study. The following anthropometric measurements were acquired: height using a stadiometer to the nearest $0.01 \mathrm{~cm}$, weight using an electronic weighing scale to the nearest $0.05 \mathrm{~kg}$, body mass index (formula) and true leg length in centimeters (measured from the anterior superior iliac spine to medial malleolus).

Males and females aged 20-69 years old were included in the study. The exclusion criteria were as follows: presence of cardiac, pulmonary, orthopedic, neurologic or rheumatic disease which prevented the participants from walking at least 10 meters without pain, previous surgery of the lower extremity that could limit ambulation, presence of lower limb deformity, use of assistive devices or lower extremity orthoses, presence of significant balance problem as assessed by standing stork test and blindness.

\section{Gait Analysis}

All data collections were conducted at the Human Performance Laboratory. The Vicon motion capture system was used to record spatiotemporal gait parameters. The 39 reflectors for motion analysis were placed on landmarks based on the study of Clark, Barnes, Holton, Summers, Stratton, 2016. Proper calibration of the instrument was done before the gait recording of each subject. Gait recording was done using the $8 \mathrm{~m}$ walkway with ten trials. All data were uploaded into the Vicon Nexus software. These were directly imported to Microsoft Excel.

\section{Statistical Analysis}

Data was encoded in a purpose built Microsoft Excel file. SPSS (version 23) was used for statistical analysis. Mean percentages and standard deviations were obtained for descriptive data and spatiotemporal gait parameters. Analysis of variance was used to determine the differences of the anthropometric data and spatiotemporal gait parameters of the different age groups. Pearson's correlation coefficient was computed to measure the strength of relationship 
between age, weight, height, body mass index, and leg length to the spatiotemporal parameters. The Eta coefficient was used to determine if a relationship exists between spatiotemporal parameters and gender which is a categorical variable. Ratio index was computed to determine symmetry of the lower extremities during gait for each spatiotemporal parameter, assuming that $X_{r}<X_{1}$, where $X_{r}$ and $X_{1}$ are the values of the right and left limbs, the factors were calculated as follows: RI (Ratio Index) $=\left(1-X_{r} / X_{l}\right)$ $x 100 \%$, where $\mathrm{RI}=0$ indicates full symmetry while $\mathrm{RI} \geq$ to 1 indicates asymmetry. [7]

Spatiotemporal parameters were divided into $10^{\text {th }}, 25^{\text {th }}, 50^{\text {th }}, 75^{\text {th }}$, and $90^{\text {th }}$ percentiles. The data was analyzed for male and female participants. Parameters were also analyzed according to age as 20-39 (young adult), 40-59 (middle adult) and 60-69 (young old) age categories. All tests of hypotheses were assessed at the $5 \%$ level of significance. A p-value of less than 0.05 was considered significant.

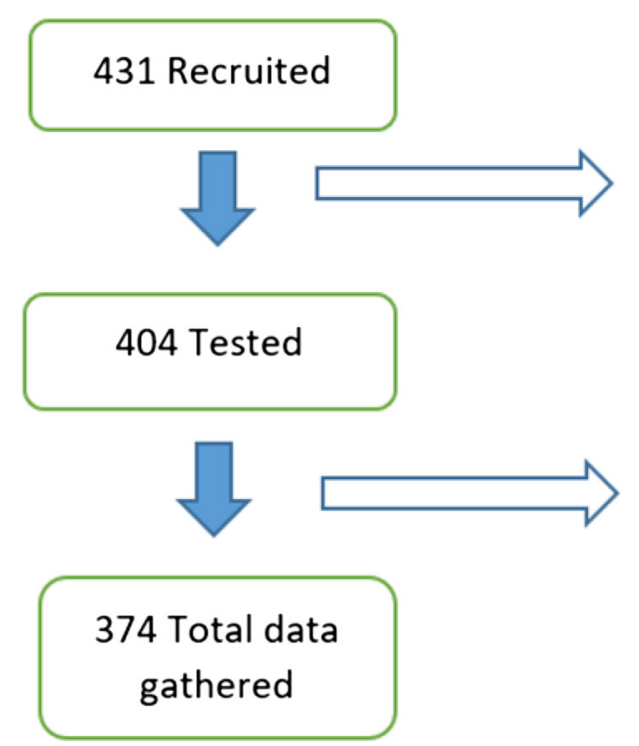

\section{RESULTS}

\section{Participants' Characteristics:}

A total of 431 participants were examined but 27 were excluded due to uncontrolled hypertension $(n=17)$, poor balance using the stork test $(n=2)$, pes planus $(n=2)$, and a history of cardiac pathology $(n=2)$, severe hallux valgus $(n=1)$, previous hip fracture $(n=1)$, previous knee fracture $(n=1)$, and peripheral arterial occlusive disease $(n=1)$. An additional 30 participants were excluded due to data error gathering giving a total of 374 participants included. (Figure 1)

Age and gender distribution of the subjects are shown in Table 1. There was an equal distribution of males and females in the YA and YO except in the MA where there were more females compared to males (55.6\% vs $44.4 \%)$.

There was a significant difference in height and leg length measurements in all participants with the YA being taller and having longer leg lengths for
27 excluded: 17 uncontrolled hypertension, 2 poor balance, 2 pes planus, 2 cardiac pathology, 1 hallux valgus, 1 hip fracture, 1 knee fracture, 1 peripheral occlusive

30 excluded due to data error gathering

Figure 1. Consort Diagram

Table 1. Age and Sex Characteristics

\begin{tabular}{lccc}
\hline \multicolumn{1}{c}{ Age group } & \multicolumn{1}{c}{ Male } & Female & Total \\
\hline $\begin{array}{l}20-39 \text { years old } \\
\text { (60\% of total population) }\end{array}$ & $114(50.2 \%$ of age group) & $113(49.8 \%$ of age group $)$ & 227 (60\% of total population) \\
$\begin{array}{l}40-59 \text { years old } \\
\text { (33\% of total population) }\end{array}$ & $56(44.4 \%$ of age group) & $70(55.6 \%$ of age group) & $126(33 \%$ of total population) \\
$\begin{array}{l}60-69 \text { years old } \\
\text { (7\% of total population) }\end{array}$ & $11(52.4 \%$ of age group) & $10(47.6 \%$ of age group) & $21(7 \%$ of total population) \\
TOTAL & $181(48.4 \%$ of total $)$ & $193(51.6 \%$ of total $)$ & $374(100 \%)$ \\
\hline
\end{tabular}


Table 2. Anthropometric characteristics according to age and gender

\begin{tabular}{|c|c|c|c|c|}
\hline All & $\begin{array}{l}\text { Age } 20 \text {-39 } \\
\text { Young adult }\end{array}$ & $\begin{array}{l}\text { Age } 40-59 \\
\text { Middle adult }\end{array}$ & $\begin{array}{l}\text { Age } 60-69 \\
\text { Young old }\end{array}$ & $P$ value \\
\hline Height (centimeters) & 159.66 & 156.27 & 156.57 & $0.00^{*}$ \\
\hline Weight (kilos) & 63.82 & 61.80 & 57.64 & 0.08 \\
\hline BMI (kilos/meter²) & 25.01 & 25.35 & 23.67 & 0.30 \\
\hline Right leg length (centimeters) & 84.60 & 82.30 & 82.48 & $0.00 *$ \\
\hline $\begin{array}{l}\text { Left leg length } \\
\text { (centimeters) }\end{array}$ & 84.46 & 82.24 & 82.45 & $0.00 *$ \\
\hline Males & Age $20-39$ & Age $40-59$ & Age $60-69$ & P value \\
\hline Height (centimeters) & 165.52 & 162.46 & 164.14 & $0.01 *$ \\
\hline Weight (kilos) & 68.95 & 66.12 & 57.23 & $0.02 *$ \\
\hline BMI (kilos/meters²) & 25.06 & 25.07 & 21.27 & $0.02^{*}$ \\
\hline Right leg length (centimeters) & 87.16 & 85.63 & 86.50 & 0.18 \\
\hline Left leg length (centimeters) & 87.08 & 85.51 & 86.36 & 0.17 \\
\hline Females & Age $20-39$ & Age $40-59$ & Age $60-69$ & P value \\
\hline Height (centimeters) & 153.74 & 151.33 & 148.75 & $0.01 *$ \\
\hline Weight (kilos) & 58.64 & 58.25 & 58.10 & 0.98 \\
\hline BMI (kilos/meters²) & 24.97 & 25.58 & 26.31 & 0.56 \\
\hline Right leg length (centimeters) & 82.02 & 79.64 & 78.05 & $0.00^{*}$ \\
\hline $\begin{array}{l}\text { Left leg length } \\
\text { (centimeters) }\end{array}$ & 81.81 & 79.62 & 78.15 & $0.00 *$ \\
\hline
\end{tabular}

*significant at 0.05 level

both extremities. These were also evident among the female participants. Among the male participants, the YA are taller and heavier when compared to the MA and YO. Body mass index was highest for the YA when compared to the YO. However, there was no statistical difference between YA and MA. There were significant differences in height, weight, and body mass index in all age groups (Table 2).

\section{Spatiotemporal Gait Parameters}

There was a significant difference in all spatiotemporal parameters between males and females. Males have statistically higher values in all spatiotemporal parameters than females except for cadence (Table 3).

There were no significant differences in the means of the following spatiotemporal parameters: step time, stride time, stance time, swing time, single limb support time, double limb support time, and cadence among all age groups. There were significant differences in the means of $Y A$ vs $Y O$ age groups and MA vs $Y O$ age groups with regard to speed and step width, where the young and middle adult age groups have significantly faster speed and greater step width than the young old age group participants. The YA, MA, and YO age groups are significantly different from each other in step length and stride length (Table 4).

YA = young adult; $M A=$ middle adult; $Y O=$ young old; NS = no significant difference

Correlation of spatiotemporal parameters with gender, age, and anthropometric measures

Age has a negative correlation with speed, step length, stride length and step width, but no correlation with step time, stride time, stance time, swing time, single and double limb support time, and cadence.

Weight has a positive correlation with step time, stride time, stance time, double limb support time, step length, stride length, and step width, but a negative correlation with cadence and no correlation with swing time, single limb support time, and speed.

Height has a positive correlation with all the gait parameters except for cadence which had a negative correlation and double limb support time which had no correlation.

Body mass index has a positive correlation with stance time, double limb support time, and step width but a negative correlation with swing time, single limb support time, speed, step length, and 
Table 3. Spatiotemporal parameters of males and females

\section{Parameters}

Sex

\begin{tabular}{ccc}
\hline Male & Female & \\
\hline $\begin{array}{c}\text { Mean + } \\
\text { Standard Deviation }\end{array}$ & Mean + & p-value \\
\hline
\end{tabular}

\begin{tabular}{lccccc}
\hline Step time (seconds) & 0.54 & 0.04 & 0.52 & 0.03 & $0.00^{*}$ \\
Stride time (seconds) & 1.09 & 0.08 & 1.05 & 0.07 & $0.00^{*}$ \\
Stance time (seconds) & 0.62 & 0.05 & 0.61 & 0.05 & $0.00^{*}$ \\
Swing time (seconds) & 0.47 & 0.03 & 0.44 & 0.03 & $0.00^{*}$ \\
Single limb support time (seconds) & 0.46 & 0.03 & 0.44 & 0.03 & $0.00^{*}$ \\
Double limb support time (seconds) & 0.08 & 0.02 & 0.08 & 0.02 & $0.00^{*}$ \\
Cadence (steps/minute) & 111.61 & 7.83 & 115.82 & 8.33 & $0.00^{*}$ \\
Speed (meters/second) & 1.15 & 0.14 & 1.12 & 0.12 & $0.00^{*}$ \\
Step length (meters) & 0.62 & 0.05 & 0.58 & 0.05 & $0.00^{*}$ \\
Stride length (meters) & 1.22 & 0.11 & 1.16 & 0.09 & $0.00^{*}$ \\
Step width (meters) & 0.09 & 0.03 & 0.08 & 0.03 & $0.00^{*}$ \\
\hline
\end{tabular}

*significant at 0.05 level

Table 4. Spatiotemporal parameters as to age groups in all participants, males and females

\begin{tabular}{|c|c|c|c|c|c|c|c|c|}
\hline \multirow[t]{3}{*}{ Parameters } & \multicolumn{8}{|c|}{ Age Category } \\
\hline & \multirow{2}{*}{\multicolumn{2}{|c|}{$\begin{array}{c}\text { 20-39 (YA) } \\
\begin{array}{c}\text { Mean } \pm \text { standard } \\
\text { deviation (SD) }\end{array}\end{array}$}} & \multirow{2}{*}{\multicolumn{2}{|c|}{$\begin{array}{c}\text { 40-59 (MA) } \\
\begin{array}{c}\text { Mean } \pm \text { standard } \\
\text { deviation (SD) }\end{array}\end{array}$}} & \multirow{2}{*}{\multicolumn{2}{|c|}{$\begin{array}{c}\text { 60-69 (YO) } \\
\begin{array}{c}\text { Mean } \pm \text { standard } \\
\text { deviation (SD) }\end{array}\end{array}$}} & \multicolumn{2}{|c|}{ ANOVA } \\
\hline & & & & & & & \multirow{2}{*}{$\begin{array}{c}\text { p-value } \\
0.84\end{array}$} & \multirow{2}{*}{$\begin{array}{c}\text { Significantly } \\
\text { different pairs } \\
\text { under DMRY } \\
\text { NS }\end{array}$} \\
\hline Step time & 0.53 & 0.04 & 0.53 & 0.04 & 0.53 & 0.03 & & \\
\hline Stride time & 1.07 & 0.07 & 1.07 & 0.08 & 1.07 & 0.07 & 0.79 & NS \\
\hline Stance time & 0.61 & 0.05 & 0.61 & 0.05 & 0.62 & 0.05 & 0.55 & NS \\
\hline Swing time & 0.45 & 0.03 & 0.45 & 0.04 & 0.45 & 0.03 & 0.98 & NS \\
\hline $\begin{array}{l}\text { Single limb support } \\
\text { time }\end{array}$ & 0.45 & 0.03 & 0.45 & 0.04 & 0.45 & 0.03 & 0.83 & NS \\
\hline $\begin{array}{l}\text { Double limb support } \\
\text { time }\end{array}$ & 0.08 & 0.02 & 0.08 & 0.02 & 0.09 & 0.02 & 0.14 & NS \\
\hline Cadence & 113.65 & 7.79 & 114.04 & 9.28 & 112.86 & 7.29 & 0.65 & NS \\
\hline Speed & 1.15 & 0.13 & 1.12 & 0.13 & 1.06 & 0.13 & 0.00 & $\begin{array}{l}\text { YA vs. YO; } \\
\text { MA vs. YO }\end{array}$ \\
\hline Step length & 0.61 & 0.05 & 0.59 & 0.05 & 0.56 & 0.06 & 0.00 & $\begin{array}{l}\text { YA vs MA; } \\
\text { MA vs. YO; } \\
\text { YA vs. YO }\end{array}$ \\
\hline Stride length & 1.21 & 0.10 & 1.17 & 0.10 & 1.12 & 0.11 & 0.00 & $\begin{array}{l}\text { YA vs. MA; } \\
\text { MA vs. YO; } \\
\text { YA vs. YO }\end{array}$ \\
\hline Step width & 0.09 & 0.03 & 0.09 & 0.03 & 0.08 & 0.04 & 0.00 & $\begin{array}{l}\text { YA vs. YO; MA } \\
\text { vs. YO }\end{array}$ \\
\hline
\end{tabular}

$Y A=$ young adult; $M A=$ middle adult; $Y O=$ young old; $N S=$ no significant difference

stride length and no correlation with step time, stride time, and cadence.

Lastly, true leg length has a positive correlation with step time, stride time, stance time, swing time, single limb support time, step length, stride length and step width but a negative correlation with cadence and no correlation with double limb support time and speed (Table 5). 
Using the Eta coefficient, gender is a significant variable for all spatiotemporal parameters of gait except for speed. Males demonstrated a longer step time, stride time, stance time, swing time, and single limb support time and longer step length, stride length, and step width than females. Females had a significantly higher cadence as compared to males (Table 6).

The ratio index was computed to determine symmetry of the right and left lower extremities. Minimal asymmetry was noted between the right and left lower extremity in stance time, double limb support time, step width, and step length in the MA and $Y O$ age groups ranging from 0.01 to 0.20 . Minimal asymmetry was likewise noted in swing time and speed in all age groups ranging from
0.01 to 0.02 . Step time, stride time, and single limb support were minimally asymmetric as well among the female $Y O$ age group ranging from 0.01 to 0.02. Cadence and stride length were symmetrical in all participants.

The mean and percentile rank of spatiotemporal parameters per gender and age group were included as a supplementary file.

\section{DISCUSSION}

This study, to our knowledge, is the first descriptive statistics for reference data of 11 spatiotemporal parameters namely: step time, stride time, stance time, swing time, single limb support time, double limb support time, cadence, speed, step length,

Table 5. Pearson's correlation of gait parameters with age and anthropometric measures

\begin{tabular}{|c|c|c|c|c|c|c|c|}
\hline & & Age & $\begin{array}{l}\text { Weight } \\
\text { (kg) }\end{array}$ & Height $(\mathrm{cm})$ & BMI & $\begin{array}{l}\text { R true leg } \\
\text { length }\end{array}$ & $\begin{array}{l}\text { L true leg } \\
\text { length }\end{array}$ \\
\hline \multirow[t]{3}{*}{ R Step time } & Pearson $r$ & .009 & $.142^{* *}$ & $.262^{* *}$ & .017 & $.314^{* *}$ & $.325^{\star *}$ \\
\hline & $p$-value & .862 & .006 & .000 & .742 & .000 & .000 \\
\hline & $N$ & 374 & 374 & 374 & 374 & 374 & 374 \\
\hline \multirow[t]{3}{*}{ R Stride time } & Pearson $r$ & .017 & $.173^{* *}$ & $.289^{* *}$ & .033 & $.329^{* *}$ & $.335^{* \star}$ \\
\hline & $p$-value & .748 & .001 & .000 & .526 & .000 & .000 \\
\hline & $N$ & 374 & 374 & 374 & 374 & 374 & 374 \\
\hline \multirow[t]{3}{*}{ R Stance time } & Pearson $r$ & .026 & $.205^{* *}$ & $.183^{* *}$ & $.137^{* *}$ & $.248^{* *}$ & $.258^{* *}$ \\
\hline & $p$-value & .621 & .000 & .000 & .008 & .000 & .000 \\
\hline & $N$ & 374 & 374 & 374 & 374 & 374 & 374 \\
\hline \multirow[t]{3}{*}{ R Swing time } & Pearson $r$ & -.002 & .071 & $.352^{* *}$ & $-.129^{\star}$ & $.343^{* *}$ & $.342^{\star \star}$ \\
\hline & $p$-value & .973 & .168 & .000 & .012 & .000 & .000 \\
\hline & $N$ & 374 & 374 & 374 & 374 & 374 & 374 \\
\hline \multirow{3}{*}{$\begin{array}{l}\text { R Single limb } \\
\text { support time }\end{array}$} & Pearson $r$ & -.011 & .037 & $.330^{* *}$ & $-.151^{\star \star}$ & $.322^{* *}$ & $.332^{* \star}$ \\
\hline & $p$-value & .825 & .471 & .000 & .003 & .000 & .000 \\
\hline & $N$ & 374 & 374 & 374 & 374 & 374 & 374 \\
\hline \multirow{3}{*}{$\begin{array}{l}\text { R Double limb } \\
\text { support time }\end{array}$} & Pearson $r$ & .033 & $.199^{\star \star}$ & -.023 & $.256^{* *}$ & .082 & .085 \\
\hline & $p$-value & .522 & .000 & .660 & .000 & .113 & .102 \\
\hline & $N$ & 374 & 374 & 374 & 374 & 374 & 374 \\
\hline \multirow[t]{3}{*}{ R Cadence } & Pearson $r$ & .024 & $-.128^{*}$ & $-.266^{\star *}$ & .002 & $-.316^{* *}$ & $-.325^{\star \star}$ \\
\hline & $p$-value & .650 & .013 & .000 & .965 & .000 & .000 \\
\hline & $N$ & 374 & 374 & 374 & 374 & 374 & 374 \\
\hline \multirow[t]{3}{*}{ R Speed } & Pearson $r$ & $-.192^{\star *}$ & -.014 & $.166^{* *}$ & $-.131^{\star}$ & .075 & .083 \\
\hline & $p$-value & .000 & .794 & .001 & .011 & .146 & .110 \\
\hline & $N$ & 374 & 374 & 374 & 374 & 374 & 374 \\
\hline \multirow[t]{3}{*}{ R Step length } & Pearson $r$ & $-.242^{* \star}$ & $.103^{*}$ & $.413^{* *}$ & $-.140^{\star *}$ & $.320^{* *}$ & $.336^{* *}$ \\
\hline & $p$-value & .000 & .046 & .000 & .007 & .000 & .000 \\
\hline & $N$ & 374 & 374 & 374 & 374 & 374 & 374 \\
\hline
\end{tabular}


Table 5. Pearson's correlation of gait parameters with age and anthropometric measures

\begin{tabular}{|c|c|c|c|c|c|c|c|}
\hline & & Age & $\begin{array}{l}\text { Weight } \\
\text { (kg) }\end{array}$ & Height (cm) & BMI & $\begin{array}{l}\text { R true leg } \\
\text { length }\end{array}$ & $\begin{array}{l}\text { L true leg } \\
\text { length }\end{array}$ \\
\hline \multirow[t]{3}{*}{ R Stride length } & Pearson $r$ & $-.247^{* *}$ & $.129^{*}$ & $.432^{* \star}$ & $-.121^{*}$ & $.347^{* \star}$ & $.364^{* *}$ \\
\hline & $p$-value & .000 & .012 & .000 & .019 & .000 & .000 \\
\hline & $N$ & 374 & 374 & 374 & 374 & 374 & 374 \\
\hline \multirow[t]{3}{*}{ R Step width } & Pearson $r$ & $-.157^{\star *}$ & $.343^{* *}$ & $.136^{* *}$ & $.316^{* *}$ & .082 & .084 \\
\hline & $p$-value & .002 & .000 & .008 & .000 & .112 & .104 \\
\hline & $N$ & 374 & 374 & 374 & 374 & 374 & 374 \\
\hline \multirow[t]{3}{*}{ L Step time } & Pearson $r$ & .014 & $.184^{* *}$ & $.288^{* *}$ & .043 & $.324^{* *}$ & $.327^{* *}$ \\
\hline & $p$-value & .786 & .000 & .000 & .402 & .000 & .000 \\
\hline & $N$ & 374 & 374 & 374 & 374 & 374 & 374 \\
\hline \multirow[t]{3}{*}{ L Stride time } & Pearson $r$ & .029 & $.177^{* *}$ & $.282^{* *}$ & .042 & $.322^{* *}$ & $.329^{* *}$ \\
\hline & $p$-value & .573 & .001 & .000 & .421 & .000 & .000 \\
\hline & $N$ & 374 & 374 & 374 & 374 & 374 & 374 \\
\hline \multirow[t]{3}{*}{ L Stance time } & Pearson $r$ & .031 & $.218^{* *}$ & $.190^{* *}$ & $.145^{\star *}$ & $.255^{\star *}$ & $.259^{* \star}$ \\
\hline & $p$-value & .545 & .000 & .000 & .005 & .000 & .000 \\
\hline & $N$ & 374 & 374 & 374 & 374 & 374 & 374 \\
\hline \multirow[t]{3}{*}{ L Swing time } & Pearson $r$ & .017 & .062 & $.343^{\star *}$ & $-.130^{\star}$ & $.332^{* *}$ & $.343^{* *}$ \\
\hline & $p$-value & .737 & .231 & .000 & .012 & .000 & .000 \\
\hline & $N$ & 374 & 374 & 374 & 374 & 374 & 374 \\
\hline \multirow{3}{*}{$\begin{array}{l}\text { L Single limb } \\
\text { support time }\end{array}$} & Pearson $r$ & -.003 & .077 & $.359^{* *}$ & $-.125^{\star}$ & $.351^{\star *}$ & $.352^{* *}$ \\
\hline & $p$-value & .960 & .139 & .000 & .016 & .000 & .000 \\
\hline & $N$ & 374 & 374 & 374 & 374 & 374 & 374 \\
\hline \multirow{3}{*}{$\begin{array}{l}\text { L Double limb } \\
\text { support time }\end{array}$} & Pearson $r$ & .029 & $.207^{* *}$ & -.053 & $.274^{\star *}$ & .024 & .027 \\
\hline & $p$-value & .574 & .000 & .306 & .000 & .649 & .601 \\
\hline & $N$ & 374 & 374 & 374 & 374 & 374 & 374 \\
\hline \multirow[t]{3}{*}{ L Cadence } & Pearson $r$ & -.008 & $-.192^{* *}$ & $-.299^{* *}$ & -.047 & $-.329^{* *}$ & $-.332^{* *}$ \\
\hline & $p$-value & .884 & .000 & .000 & .360 & .000 & .000 \\
\hline & $N$ & 374 & 374 & 374 & 374 & 374 & 374 \\
\hline \multirow[t]{3}{*}{ L Speed } & Pearson $r$ & $-.197^{* *}$ & -.013 & $.142^{* *}$ & $-.109^{*}$ & .074 & .083 \\
\hline & $p$-value & .000 & .797 & .006 & .034 & .152 & .108 \\
\hline & $N$ & 374 & 374 & 374 & 374 & 374 & 374 \\
\hline \multirow[t]{3}{*}{ L Step length } & Pearson $r$ & $-.247^{\star \star}$ & $.134^{* *}$ & $.424^{* *}$ & $-.107^{*}$ & $.362^{* *}$ & $.375^{\star *}$ \\
\hline & $p$-value & .000 & .010 & .000 & .039 & .000 & .000 \\
\hline & $N$ & 374 & 374 & 374 & 374 & 374 & 374 \\
\hline \multirow[t]{3}{*}{ L Stride length } & Pearson $r$ & $-.242^{\star \star}$ & $.137^{* *}$ & $.433^{* *}$ & $-.111^{*}$ & $.353^{* *}$ & $.367^{* *}$ \\
\hline & $p$-value & .000 & .008 & .000 & .032 & .000 & .000 \\
\hline & $N$ & 374 & 374 & 374 & 374 & 374 & 374 \\
\hline \multirow[t]{3}{*}{ L Step width } & Pearson $r$ & -.096 & $.315^{* *}$ & $.155^{* *}$ & $.278^{* *}$ & .094 & $.102^{*}$ \\
\hline & $p$-value & .063 & .000 & .003 & .000 & .069 & .050 \\
\hline & $N$ & 374 & 374 & 374 & 374 & 374 & 374 \\
\hline
\end{tabular}

$R=$ right; $L=$ left; ${ }^{*}$ significant at 0.05 level; ${ }^{*}$ significant at 0.01 level 
Table 6. Eta Coefficient of sex with gait parameters

\begin{tabular}{lll}
\hline & Eta & p-value \\
\hline R Step time * Sex & .240 & 0.000 * \\
R Stride time * Sex & .260 & 0.000 * \\
R Stance time * Sex & .111 & $0.029 *$ \\
R Swing time * Sex & .398 & 0.000 * \\
R Single limb support time * Sex & .379 & $0.000 *$ \\
R Double limb support time * Sex & .133 & $0.009 *$ \\
R Cadence * Sex & .240 & $0.000 *$ \\
R Speed * Sex & .115 & $0.023 *$ \\
R Step length * Sex & .315 & $0.000 *$ \\
R Stride length * Sex & .327 & $0.000 *$ \\
R Step width * Sex & .112 & $0.028 *$ \\
L Step time * Sex & .264 & $0.000 *$ \\
L Stride time * Sex & .252 & $0.000)$ \\
L Stance time * Sex & .132 & $0.009 *$ \\
L Swing time * Sex & .367 & $0.000 *$ \\
L Single limb support time * Sex & .398 & $0.000 *$ \\
L Double limb support time * Sex & .149 & $0.003 *$ \\
L Cadence * Sex & .267 & $0.000 *$ \\
L Speed * Sex & .092 & 0.069 \\
L Step length * Sex & .331 & $0.000 *$ \\
L Stride length * Sex & .336 & $0.000 *$ \\
L Step width * Sex & .205 & 0.000 \\
\hline * & &
\end{tabular}

${ }^{*}$ significant at 0.05 level

stride length, and step width, according to age and gender in percentile ranking and is the first known reference data on gait among Filipinos. These data could provide clinicians and researchers values against which measurements can be compared for assessing and interpreting gait dysfunction. [8]

In comparison to the Filipino data we have gathered, the French have the fastest walking speed at $144 \mathrm{~cm} / \mathrm{min}$ followed by the Americans at 130.6 $\mathrm{cm} / \mathrm{min}$, then the Italians at $125.6 \mathrm{~cm} / \mathrm{min}$. Filipino women have a mean speed of $115.9 \mathrm{~cm} / \mathrm{min}$ which is a little faster than the Korean women at 112.5 $\mathrm{cm} / \mathrm{min}$, while Filipino men almost have the same walking speed as the Korean men at $111.7 \mathrm{~cm} /$ min and $111.8 \mathrm{~cm} / \mathrm{min}$, respectively. Italians have the longest stride length $(\mathrm{cm})$ at $140 \mathrm{~cm}$, followed by the French at $139 \mathrm{~cm}$ and then the Americans at $136.1 \mathrm{~cm}$. Likewise, Filipino and Korean stride length values are closer with mean value of 115.8 $\mathrm{cm}$ among Filipino women and $116.7 \mathrm{~cm}$ among Korean women and $122.5 \mathrm{~cm}$ among Filipino men and $126.6 \mathrm{~cm}$ among Korean men. Filipino women have the greatest cadence (step/min) at 115.9 followed by Korean women at 112.5. Cadence mean values in steps/min are almost equal at 111.6 for Americans, 111.8 for Korean men and 111.7 for Filipino men with Italians having the least number of steps/minute at 105.6. The Koreans have a greater step width $(\mathrm{cm})$ with mean value of 16.7 $\mathrm{cm}$ for women and $19.4 \mathrm{~cm}$ for men as compared to only $8.3 \mathrm{~cm}$ and $9.0 \mathrm{~cm}$ for Filipino females and males, respectively (Table 7). The stride length and walking speed of Filipinos and Koreans are significantly smaller and slower than Americans and Caucasians. This difference is thought to be affected by the stature difference between race because taller people have longer stride lengths and stride length is strongly correlated with walking speed.[9]

Walking speed, step length, stride length and step width decline significantly with age. Absolute values for all the above parameters were higher in men than women at all ages. These finding of decline in walking speed values and stride length as you age also confers with the findings of Samson, et al. and Buchner, et al.[10,1 1]

With regard to gender our study showed that all spatiotemporal parameters namely step time, stride time, stance time, swing time, single limb support time, double limb support time, speed, step length, stride length, and step width were significantly increased in men, except for cadence which was significantly increased in women. These findings were also in accordance with the studies by Callisaya, Blizzard, Schmidt, McGinley \& Srikanth [12] where mean cadence for men was 107.2 steps/min and women was 114.6 steps/min, stride length of 130 $\mathrm{cm}$ for men and $115 \mathrm{~cm}$ for women, step time of 0.56 for men and 0.53 for women, step width of 10 $\mathrm{cm}$ for men and $7.9 \mathrm{~cm}$ for women. According to Hollmann, et al. [8], men tended to walk with longer strides but with lower cadence. This could further be explained by the fact that the women's physique is significantly smaller than men in terms of height and leg length that it takes more steps to cover a certain distance by women participants as compared to the men.[13]

The body mass index in our study has a positive correlation with stance time, double limb support time, and step width and a negative correlation with swing time, single limb support time, speed, step length, and stride length and no significant correlation with 
Table 7. Comparison of the spatiotemporal parameters of studies from different countries

\begin{tabular}{|c|c|c|c|c|c|}
\hline Parameters & $\begin{array}{l}\text { Our study } \\
\text { Philippines }\end{array}$ & $\begin{array}{c}\text { Kadaba (1989) } \\
\text { USA }\end{array}$ & $\begin{array}{l}\text { Auvinet (2002) } \\
\text { France }\end{array}$ & $\begin{array}{l}\text { Benedetti (1998) } \\
\text { Italy }\end{array}$ & $\begin{array}{c}\text { Cho (2004) } \\
\text { Korea }\end{array}$ \\
\hline Subjects & $\begin{array}{l}N=374 \\
\text { Age: } 20-69\end{array}$ & $\begin{array}{l}\mathrm{N}=40 \\
\text { Age: } 18-40\end{array}$ & $\begin{array}{l}N=282 \\
\text { Age: } 20-98\end{array}$ & $\begin{array}{l}N=20 \\
\text { Age: } 20-72\end{array}$ & $\begin{array}{l}\mathrm{N}=98 \\
\text { Age: early } 20 \mathrm{~s}\end{array}$ \\
\hline Cadence (steps/min) & $\begin{array}{l}130.3(\mathrm{M}) \\
111.8(\mathrm{~F})\end{array}$ & 111.6 & & $\begin{array}{l}105.6(\mathrm{M}) \\
111.6(\mathrm{~F})\end{array}$ & $\begin{array}{l}111.8(\mathrm{M}) \\
112.5(\mathrm{~F})\end{array}$ \\
\hline Speed (meters/second) & $\begin{array}{l}1.15(20-39) \\
1.11(40-59) \\
1.06(60-69)\end{array}$ & 1.306 & $\begin{array}{ll}\text { (F) } & 1.54(20-29) \\
1.56(30-39) \\
1.50(40-49) \\
1.48(50-59) \\
1.35(60-69) \\
1.26(>70) \\
\text { (M) } 1.59(20-29) \\
1.54(30-39) \\
1.63(40-49) \\
1.42(50-59) \\
1.47(60-69) \\
1.32(>70)\end{array}$ & $\begin{array}{l}1.29(\mathrm{M}) \\
1.19(\mathrm{~F})\end{array}$ & $\begin{array}{l}1.19(\mathrm{M}) \\
1.16(\mathrm{~F})\end{array}$ \\
\hline Stride length $(\mathrm{cm})$ & $\begin{array}{l}120(\mathrm{M}) \\
114(\mathrm{~F})\end{array}$ & 136.1 & $\begin{array}{ll}\text { (F) } 149(20-29) \\
150(30-39) \\
142(40-49) \\
141(50-59) \\
132(60-69) \\
125(>70) \\
\text { (M) } 165(20-29) \\
157(30-39) \\
164(40-49) \\
149(50-59) \\
153(60-69) \\
138(>70)\end{array}$ & $\begin{array}{l}146(\mathrm{M}) \\
128(\mathrm{~F})\end{array}$ & $\begin{array}{l}126.6(\mathrm{M}) \\
116.7(\mathrm{~F})\end{array}$ \\
\hline Step width $(\mathrm{cm})$ & $\begin{array}{l}9(M) \\
7(F)\end{array}$ & & & & $\begin{array}{l}19.4(\mathrm{M}) \\
16.7(\mathrm{~F})\end{array}$ \\
\hline
\end{tabular}

$M=$ males $F=$ females

step time, stride time, and cadence. These findings were in agreement with the data gathered by Lai, Leung, Li, and Zang [14] wherein they examined and compared 14 obese subjects with $\mathrm{BMI}>30 \mathrm{~kg} /$ $\mathrm{m} 2$ against 14 subjects with normal $\mathrm{BMI}$ using the Vicon 370. It showed significant difference of their means between obese and non-obese subjects with p-values $<0.01$ as follows: the mean speed among obese subjects was $1.12 \mathrm{~m} / \mathrm{s}$ which is less than the mean speed among non-obese at $1.27 \mathrm{~m} / \mathrm{s}$, mean stride length among obese was $0.71 \mathrm{~m}$ which is less than those of non-obese at $0.77 \mathrm{~m}$. There is increase in double limb support time and stance time among the obese compared to non-obese at 11.29 from 9.55 and 60.21 from 58.51, respectively. Likewise, these findings are also in accordance with the findings of McGraw, McClenaghan, William, Dickerson and Ward [15], wherein obese boys spent a significantly greater percentage of the gait cycle in double support and stance, and less time in the swing phase than did the non-obese boys. They suggested that the extended periods of support seen in obese children were indicative of underlying postural instability.

It is a fact that even healthy subjects show a certain degree of asymmetry between the right and the left side of the body.[16] For this reason the gait parameters of the lower limbs show minimal differences between the right and left lower extremities in stance time, double limb support time, step width, and step length in the MA and YO age groups. Minimal asymmetry was likewise noted in swing time and speed in all age groups. Step time, stride time, and single limb support likewise showed minimal asymmetry among the female $Y O$ age group only. All participants showed symmetry 
in cadence and stride length. These asymmetries in gait, specifically stance time, double limb support time, step width, and step length in the MA and YO age groups can be attributed to the elderly people's attempt for greater stability and balance in walking.

\section{CONCLUSION}

The authors have presented a reference data for spatiotemporal parameters among healthy Filipinos aged 20-69 years. Our study has shown the correlation of the different spatiotemporal parameters with age, gender, and anthropometric measures. Likewise, minimal asymmetry was observed in the 40-59 and 60-69 age groups stance time, double limb support time, step width, and step length. This reference data, to our knowledge, is the first study done using Filipino participants which could serve as a reference for gait studies in the Philippines.

\section{Implications for Physiatric Practice}

This reference data of spatiotemporal gait parameters among healthy Filipinos aged 20-69 years is an extremely useful source for physiatrists in the Philippines involved in measuring and analyzing gait of their patients. Clinically relevant variables in their patient's gait can be compared to this normative reference data to assist understanding of its pathophysiology so that a more appropriate therapeutic approach can be made to address their patient's gait problems.

\section{Limitation of the study}

The study had a relatively fewer participants in the young, old, and none in the other old age groups because the number of participants recruited per age group was based on the percentage of the population in the city of Manila. Further studies on gait analysis should be performed not only for the older age population but for the pediatric population as well.

\section{Conflict of Interest and Source of Funding:}

Funding was provided by the Philippine Council for Health Research and Development - Department of Science and Technology (PCHRD-DOST). The investigators have no conflicts of interest in any form with the sponsor or the study. 


\section{REFERENCES}

1. Bugané $F$, Benedetti $M G$, Casadio $G$, Attala $S$, Biagi $F$, Manca $M$, et al. Estimation of spatial-temporal gait parameters in level walking based on a single accelerometer: validation on normal subjects by standard gait analysis. Comput Methods Programs Biomed [Internet]. 2012;108(1):129-37. Available from: http://dx.doi. org/10.1016/i.cmpb.2012.02.003

2. Al-Obaidi S, Wall JC, Al-Yaqoub A, Al-Ghanim M. Basic gait parameters: a comparison of reference data for normal subjects 20 to 29 years of age from Kuwait and Scandinavia. J Rehabil Res Dev [Internet]. 2003;40(4):361-6. Available from: http://dx.doi.org/10.1682/jrrd.2003.07.0361

3. Patterson KK, Nadkarni NK, Black SE, Mcllroy WE. Gait symmetry and velocity differ in their relationship to age. Gait Posture [Internet]. 2012;35(4):590-4. Available from: http://dx.doi.org/10.1016/i.gaitpost.2011.11.030

4. Kwon JW, Son SM, Lee NK. Changes of kinematic parameters of lower extremities with gait speed: a 3D motion analysis study. J Phys Ther Sci [Internet]. 2015;27(2):477-9. Available from: http://dx.doi.org/10.1589/ipts.27.477

5. Majernik J. Normative human gait databases. Statistics Research Letters. 2013;2:69-74. Available from: https://www.academia.edu/download/48138330/ SRL10020_2_3_69_74.pdf

6. Ryu T, Choi HS, Choi HW, Chung MK. A comparison of gait characteristics between Korean and Western people for establishing Korean gait reference data. Int J Ind Ergon. 2006;36:1023-30. Available from: https://www.sciencedirect.com/science/article/pii/S0169814106001818

7. Blazkiewicz M, Wiszomirska I, Wit A. Comparison of four methods of calculating the symmetry of spatiotemporal parameters of gait. Acta Bioeng Biomech. 2014;16:29-35. Available from: https://yadda. icm.edu.pl/baztech/element/bwmetal . element. baztech-87c71492-aaec-4ba5-b8ab-945b161a9374

8. Hollman JH, MacDade EM, Petersen RC. Normative spatiotemporal gait parameters in adults, Gait Posture. $2011 ; 34: 111-8$. Available from: https://www.sciencedirect.com/science/article/pii/S0966636211001019

9. Pierryrowski MR, Galea V. Enhancing the ability of gait analyses to differentiate between groups: scaling gait data to body size. Gait Posture. $2001 ; 13: 193-201$. Available from: https://www.sciencedirect.com/science/article/pii/ S0966636201000972

10. Samson MM, Crowe A, de Vreede PL, Dessens JA, Duursma SA, Verhaar HJ. Difference in gait parameters at a preferred walking speed in healthy subjects due to age, height and body weight. Aging Clin. Exp. Res. 2001;13:16-21. Available from: https://link.springer. com/article/10.1007/BF03351489
11. Buchner D, Cress M, Esselman P, Margherita A, de Lateur B, Campbell V, et al. Factors associated with changes in gait speed in older adults. J Gerontol. 1996;51 A:297-302. Available from: https://academic.oup.com/biomedgerontology/ article-abstract/51 A/6/M297/593066

12. Callisaya M, Blizzard L, Schmidt M, McGinley J, Srikanth V. Aging and Age variability. A population based study of older people. Age Ageing. 2010;39:191-7. Available from: https://academic.oup.com/ageing/ article-abstract/39/2/191/41315

13. Auvinet B, Berrut G, Touzard C, Moutel L, Collet N, Chaleil $D$, et al. Reference data for normal subjects obtained with an accelerometric device. Gait Posture. 2002;16:124-34. Available from: https://www.sciencedirect.com/science/ article/pii/S096663620100203X

14. Lai $P$, Leung A, Li A, Zhang M. Three dimensional gait analysis of obese adults. Clin Biomech. 2008;23(1):S2-S6. Available from: https://www.sciencedirect.com/science/ article/pii/S0268003308000387

15. McGraw B, McClenaghan A, William H, Dickerson J, Ward D. Gait and postural instability in obese and non-obese prepubertal boys. Arch Phys Med Rehabil. 2000;81:484-9. Available from: https://www.sciencedirect.com/science/ article/pii/S0003999300636122

16. Macellari V, Giacomozzi C, Saggini R. Spatio-temporal parameters of gait: reference data and a statistical method for normality assessment. Gait Posture. 1999;10:171-81. Available from: https://www.sciencedirect.com/science/ article/pii/S0966636299000211

(1) Open Access This article is licensed under a c) ${ }_{\text {BY NC SA }}$ Creative Commons Attribution-NonCommercialShareAlike 4.0 International License, which permits use, share - copy and redistribute the material in any medium or format, adapt - remix, transform, and build upon the material, as long as you give appropriate credit, provide a link to the license, and indicate if changes were made. You may do so in any reasonable manner, but not in any way that suggests the licensor endorses you or your use. You may not use the material for commercial purposes. If you remix, transform, or build upon the material, you must distribute your contributions under the same license as the original. You may not apply legal terms or technological measures that legally restrict others from doing anything the license permits. The images or other third party material in this article are included in the article's Creative Commons license, unless indicated otherwise in a credit line to the material. If material is not included in the article's Creative Commons license and your intended use is not permitted by statutory regulation or exceeds the permitted use, you will need to obtain permission directly from the copyright holder. To view a copy of this license, visit https://creativecommons.org/licenses/by-nc-sa/4.0/. 